\title{
Coherent manipulation of electronic states in a double quantum dot
}

\author{
T. Hayashi ${ }^{1}$, T. Fujisawa ${ }^{1}$, H. D. Cheong ${ }^{2}$, Y. H. Jeong ${ }^{3}$, and Y. Hirayama ${ }^{1,4}$ \\ ${ }^{1}$ NTT Basic Research Laboratories, NTT Corporation, \\ 3-1 Morinosato-Wakamiya, Atsugi, 243-0198, Japan \\ ${ }^{2}$ Keimyung University, Shindang-Dong, Dalseo-gu, Daegu, Korea \\ ${ }^{3}$ Pohang University of Science and Technology, Pohang, Kyungpook, Korea and \\ ${ }^{4}$ CREST, 4-1-8 Honmachi, Kawaguchi, 331-0012, Japan
}

(Dated: February 2, 2008)

\begin{abstract}
We investigate coherent time-evolution of charge states (pseudo-spin qubit) in a semiconductor double quantum dot. This fully-tunable qubit is manipulated with a high-speed voltage pulse that controls the energy and decoherence of the system. Coherent oscillations of the qubit are observed for several combinations of many-body ground and excited states of the quantum dots. Possible decoherence mechanisms in the present device are also discussed.
\end{abstract}

Initiated by various experiments on atomic systems, studies on coherent dynamics have been extended to small-scale quantum computers [1]. Nano-fabrication technology now allows us to design artificial atoms (quantum dots) and molecules (coupled quantum dots), in which atomic (molecular)- like electronic states can be controlled with external voltages [2, 3, 4]. Coherent manipulation of the electronic system in quantum dots and a clear understanding of decoherence in practical structures are crucial for future applications of quantum nanostructures to quantum information technology.

In this Letter, we describe the coherent manipulation of charge states, in which an excess electron occupies the left dot or the right dot of a double quantum dot (DQD). The coherent oscillations between the two charge states are produced by applying a rectangular voltage pulse to an electrode. Although this scheme is analogous to experiments on a superconducting island 5], our qubit is effectively isolated from the electrodes during the manipulation, while it is influenced by strong decoherence during the initialization due to the coupling with the electrodes. This controlled decoherence provides an efficient initialization scheme.

We consider a DQD consisting of left and right dots connected through an interdot tunneling barrier. The left (right) dot is weakly coupled to the source (drain) electrode via a tunneling barrier [see Fig. 1(a)]. The conductance through the device is strongly influenced by the onsite and interdot Coulomb interactions [6]. In the weakcoupling regime at a small source-drain voltage, $V_{s d}$, a finite current is only observed at the triple points, where tunneling processes through the three tunneling barriers are allowed. Under an appropriate condition where only the interdot tunneling is allowed, Coulomb interactions effectively isolate the DQD from the source and drain electrodes. In this case, we can consider two charge states, in which an excess electron occupies the left dot $(|L\rangle)$ or the right $\operatorname{dot}(|R\rangle)$ with electrochemical potentials $E_{L}$ and $E_{R}$, respectively. In practice, each charge state involves (many-body) ground and excited states. When the two specific states are energetically close to each other and the excitation to other states can be neglected, the system can be approximated as a two-level system (qubit). It is characterized by the energy offset, $\varepsilon \equiv E_{R}-E_{L}$, and the interdot tunneling, which gives an anti-crossing energy, $\Delta[3]$. The effective Hamiltonian is

$$
H=\frac{1}{2} \varepsilon(t) \sigma_{z}+\frac{1}{2} \Delta \sigma_{x}
$$

where $\sigma_{x}$ and $\sigma_{z}$ are the Pauli matrices for pseudo-spin bases of $|L\rangle$ and $|R\rangle$. When $E_{L}$ and $E_{R}$ of the localized states are crossed by changing $V_{s d}$, for instance, as shown by dashed lines in Fig. 1(b), the eigenenergies, $E_{b}$ and $E_{a}$, for bonding and anti-bonding states respectively, show anti-crossing as shown by solid lines. The coherent oscillation of the system is expected with the angular frequency given by $\Omega=\sqrt{\varepsilon^{2}+\Delta^{2}} / \hbar$.

The DQDs (samples I and II with almost identical dimensions) used for this work are defined in a GaAs/AlGaAs heterostructure containing a twodimensional electron gas, as shown in Fig. 1(a). The experiments were performed in a magnetic field of $0.5 \mathrm{~T}$ at lattice temperature $T_{\text {lat }} \lesssim 20 \mathrm{mK}$, unless otherwise noted. The effective electron temperature, however, remained at $T_{\text {elec }} \sim 100 \mathrm{mK}$. Each dot in both samples contains about 25 electrons and has an on-site charging energy $E_{c} \sim 1.3 \mathrm{meV}$. The interdot electrostatic coupling energy is $U \sim 200 \mu \mathrm{eV}$. Figure 2(a) shows the current spectrum $I$ of sample I when the voltage, $V_{R}$, on the right gate $\left[G_{R}\right.$ in Fig $\left.1(\mathrm{a})\right]$ is swept at a large source-drain voltage $V_{s d}=650 \mu \mathrm{V}$. Each dot contains several energy states in the transport window of width $e V_{s d}$, and resonant tunneling between them is clearly resolved as current peaks, two of which (resonances $\alpha$ and $\beta$ ) are shown in Fig. 2(a). Resonance $\alpha(\beta)$ is probably associated with the ground state of the left dot and the first (second) excited state of the right dot. In the vicinity of each peak, a two-level system (qubit) can be defined by only taking into account a single discrete state in each dot, $|L\rangle$ and $|R\rangle$. The qubit parameters, $\varepsilon$ and $\Delta$, and tunneling rates, $\Gamma_{L}$ and $\Gamma_{R}$, respectively for left and right barriers, can be controlled independently by exter- 
(a)

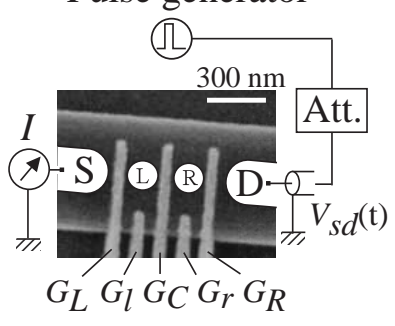

(b)

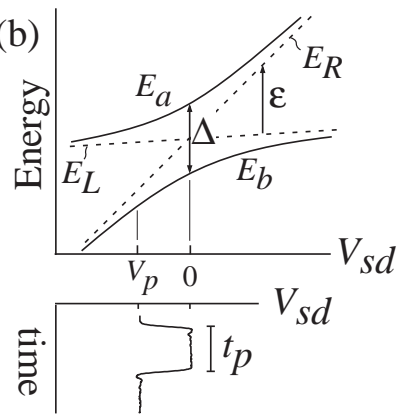

(c) initialization

$$
\begin{aligned}
& V_{s d}=V_{p} \\
& \varepsilon=\varepsilon_{0}<0
\end{aligned}
$$

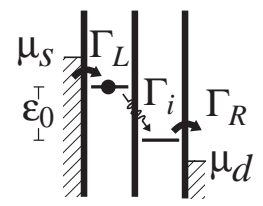

(d) manipulation

$$
\begin{aligned}
& V_{s d}=0 \\
& \varepsilon=\varepsilon_{1}=0
\end{aligned}
$$

(e) measurement

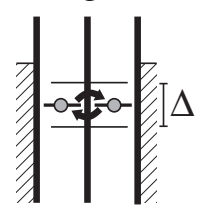

$V_{s d}=V_{p}$ $\varepsilon=\varepsilon_{0}$

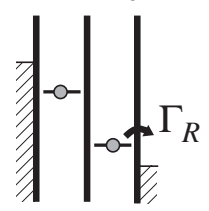

FIG. 1: (a) Schematic measurement circuit combined with a scanning electron microscope image of the sample. Etching (upper and lower dark regions) and negatively biased gate electrodes $\left(G_{L}, G_{l}, G_{C}, G_{r}\right.$, and $\left.G_{R}\right)$ define a double quantum dot (L and R) between the source (S) and drain (D). (b) Energy levels of the bonding $\left(E_{b}\right)$ and anti-bonding $\left(E_{a}\right)$ states, which are the eigenstates during the manipulation, and localized states $\left(E_{L}\right.$ and $\left.E_{R}\right)$ during initialization. A typical condition of $\varepsilon_{1}=0$, where $E_{L}$ and $E_{R}$ cross at $V_{s d}=0$, is shown. A typical pulsed voltage $V_{s d}(t)$ is shown at the bottom. (c) - (e) Energy diagrams of the DQD for $\varepsilon_{1}=0$ during (c) initialization, (d) coherent oscillation, and (e) measurement process.

nal gate voltages, and are determined from the elastic current spectra $[3,4,6]$.

In order to manipulate the qubit, a rectangular voltage pulse is applied to the drain electrode. This switches the source-drain bias voltage $V_{s d}$ between $V_{p}=650 \mu \mathrm{V}$, at which the tunneling between the DQD and the electrodes is allowed, and zero, at which the DQD is effectively isolated from the electrode due to Coulomb interactions. At the same time, due to the electrostatic coupling between the QDs and electrodes, the pulse also switches the energy offset between $\varepsilon=\varepsilon_{0}$ at $V_{s d}=V_{p}$ and $\varepsilon_{1}$ at $V_{s d}=0$ $\left(\varepsilon_{1}-\varepsilon_{0} \sim 30 \mu \mathrm{eV}\right)$, as shown for $\varepsilon_{1}=0$ in Fig. 1(b). We designed the pulse sequence for initialization, coherent manipulation, and measurement in the following way.

For initialization, a relatively large source-drain voltage, $V_{s d}=V_{p}$, was applied under appropriate gate voltages, so that $E_{L}$ and $E_{R}$ are in between the electrochemical potentials of the source and drain electrodes, $\mu_{S}$ and $\mu_{D}\left(\mu_{S}>E_{L}, E_{R}>\mu_{D}=\mu_{S}-e V_{p}\right)$. For example, in the off-resonance condition $\left(\varepsilon=\varepsilon_{0} \lesssim-\Delta\right)$ as shown in Fig. 1(c), electron-phonon interaction provides finite inelastic tunneling, whose rate is $\Gamma_{i}$, between the two states [4]. We adjusted $\Gamma_{L}$ and $\Gamma_{R}$ to make them sufficiently

larger than $\Gamma_{i}$ so that the current would be limited by the inelastic tunneling between the dots. This sequential tunneling process accumulates an excess electron in the left dot, providing the initial state $|L\rangle$. Note that this initialization works even in the resonance condition $\left(\varepsilon_{0}=0\right)$ when $\hbar \Gamma_{L}$ and $\hbar \Gamma_{R}$ are greater than $\Delta$. Significant decoherence from the dissipative tunneling processes holds the system in the localized state $|L\rangle$ rather than in the delocalized states.

For coherent manipulation, we non-adiabatically change $V_{s d}$ to zero, which shifts the energy offset to $\varepsilon=\varepsilon_{1}$. A typical energy diagram for $\varepsilon_{1} \sim 0$ is shown in Fig. 1(d). In this case, the inter-dot electrostatic coupling prevents the electron tunneling into and out of the DQD by any first-order tunneling process, and negligible current flows through the DQD. Hence the system is well approximated by Eq. 1 The system prepared in $|L\rangle$ goes back and forth between $|L\rangle$ and $|R\rangle$ coherently. We maintain $V_{s d}=0$ for the pulse length, $t_{p}=80-2000 \mathrm{ps}$, during which the oscillation continues.

Then, the large bias voltage is restored for the measurement [Fig. 1(e)]. The large tunneling rates $\left(\hbar \Gamma_{L}\right.$, $\hbar \Gamma_{R}>\Delta$ ) effectively stop the coherent manipulation, and thereby provide a strong measurement. If the system ends up in $|R\rangle$ after the manipulation, the electron tunnels out to the drain electrode and contributes to the pumping current. The system goes back to the initial state $|L\rangle$ after waiting longer than $\Gamma_{L}^{-1}+\Gamma_{R}^{-1}$. However, no pumping current is expected for $|L\rangle$, which is already the initial state. Hence, this pumping current depends on the probability of finding the system in $|R\rangle$.

In practice, we repeatedly applied many pulses with a repetition frequency $f_{\text {rep }}=100 \mathrm{MHz}$ and measured the average dc current, $I$, which comprises the coherent pumping current and inelastic current that flows during initialization. In order to improve the signal-to-noise ratio, we employed a lock-in amplifier technique to measure the pulse-induced current $I_{p}$ by switching the pulse train on and off at a low modulation frequency of $100 \mathrm{~Hz}$. We estimated the average number of pulse-induced tunneling electrons, $n_{p}=I_{p} / e f_{r e p}$.

A color plot of $n_{p}$ as functions of $V_{R}$ and $t_{p}$ is shown in Fig. 2(b). Sweeping $V_{R}$ mainly shifts $E_{R}$ and changes the energies $\varepsilon_{0}$ and $\varepsilon_{1}$ simultaneously by keeping $\varepsilon_{1}-\varepsilon_{0}$ almost constant. A clear oscillation pattern is observed in a wide range of $V_{R}$. Local-maxima of the oscillation amplitude appeared for relatively long $t_{p}$ at gate voltages indicated by long-dashed lines, where the two states must be resonant $\left(\varepsilon_{1}=0\right)$ during manipulation. We confirmed that the oscillation patterns in Fig. 2(b) are attributed to resonances $\alpha$ (clear oscillation) and $\beta$ (faint oscillation) from their $V_{p}$ dependence. The energy offsets $\varepsilon_{0, i}$ and $\varepsilon_{1, i}$ for resonance $i$ ( $\alpha$ and $\beta$ ) are also shown in Fig. 2(b).

The oscillation pattern for resonance $\alpha$ shows that the amplitude and period decrease as $\varepsilon_{1, \alpha}$ goes away from $\varepsilon_{1, \alpha}=0$. The current amplitude is asymmetric about 
$\varepsilon_{1, \alpha}=0$, and the oscillation continues until $\varepsilon_{1, \alpha} \sim$ $40 \mu \mathrm{eV}$. These features are qualitatively consistent with a calculation based on the time-dependent Schrödinger equation and Eq. 1 using a time-dependent $\varepsilon(t)$ with a finite rise time $(\sim 100 \mathrm{ps})$ of the pulse [5]. The strong oscillation in the range of $\varepsilon_{0, \alpha}<0<\varepsilon_{1, \alpha}$ can be understood as an interference between coherent time-evolution at $\varepsilon(t) \sim 0$ during the finite rise time of the pulse and that during the fall time of the pulse. It should be noted that clear oscillation is seen even at $\varepsilon_{0, \alpha}=0$ (indicated by a black dotted line), where two localized states are resonant during the initialization but off-resonant during the manipulation. This feature is convincing evidence that there is strong decoherence during initialization. The density matrix calculation for our initialization condition gives the decoherence rate, $\hbar\left(\Gamma_{R}+\Gamma_{L}\right) / 2 \sim 30 \mu \mathrm{eV}$ [7], which is greater than $\Delta=9 \mu \mathrm{eV}$ for resonance $\alpha$. However, the Coulomb blockade effect eliminates this decoherence during manipulation, as mentioned before. Therefore, we presume that the oscillation at $\varepsilon_{0, \alpha}=0$ is induced by the modulation of the decoherence rate. In contrast, the disappearance of the oscillation at $\varepsilon_{0, \beta}=0$ (indicated by a white dotted line) for resonance $\beta(\Delta=30 \mu \mathrm{eV})$ might arise from the inefficient initialization that provides a statistical mixture of bonding and antibonding states.

The qubit state can be manipulated arbitrarily. Ideally, the quarter period oscillation at $\varepsilon_{1}=0$ corresponds to the $\pi / 2$ pulse that prepares a superposition state $\frac{1}{\sqrt{2}}(|L\rangle+i|R\rangle)$. Leaving a state at $\varepsilon=\varepsilon_{2} \gg \Delta$ for a specific time $t_{\phi}$ gives a phase shift $\varepsilon_{2} t_{\phi} / \hbar$ between $|L\rangle$ and $|R\rangle$. Therefore arbitrary states can be prepared by tailoring the pulse waveform $\varepsilon(t)$ even at a constant $\Delta$. The demonstration of phase-shift operations will be published elsewhere [8].

Figure $2(\mathrm{c})$ shows typical $n_{p}\left(t_{p}\right)$ traces at $\varepsilon_{1}=0$ for resonances $\alpha$ and $\beta$. The oscillation can be fitted well by an exponential decay of the cosine function and a linearly decreasing term,

$$
n_{p}\left(t_{p}\right) \simeq A-\frac{1}{2} B \exp \left(-t_{p} / T_{2}\right) \cos \left(\Omega t_{p}\right)-\Gamma_{i} t_{p},
$$

except when $t_{p} \lesssim 100 \mathrm{ps}$ (the rise time of the pulse). The last term comes from the fact that the inelastic tunneling current is blocked during the manipulation. Actually $\Gamma_{i} \sim(6 \mathrm{~ns})^{-1}$ obtained for $\alpha$ from this fitting is consistent with the inelastic dc current, which should be $e \Gamma_{i}$ in the absence of the pulse. The offset, $A \sim 0.6$, and amplitude, $B \sim 0.3$, of the oscillation for $\alpha$ are comparable to the ideal case $\left(A=0.5\right.$ and $B=1$ at $\left.\varepsilon_{1}=0\right)$, although they are degraded by the finite rise time of the pulse and non-ideal initialization/measurement processes. The oscillation frequency $\Omega$ and the decoherence time $T_{2}$ can be obtained from the fitting $\left(\Omega / 2 \pi \sim 2.3 \mathrm{GHz}\right.$ and $T_{2} \sim 1$ ns for resonance $\alpha$ at $\left.\varepsilon_{1}=0\right)$.

We estimate how the decoherence rate $T_{2}^{-1}$ depends on the energy offset $\varepsilon_{1}$ [Fig. 3(a)], coupling energy $\Delta$ [Fig.

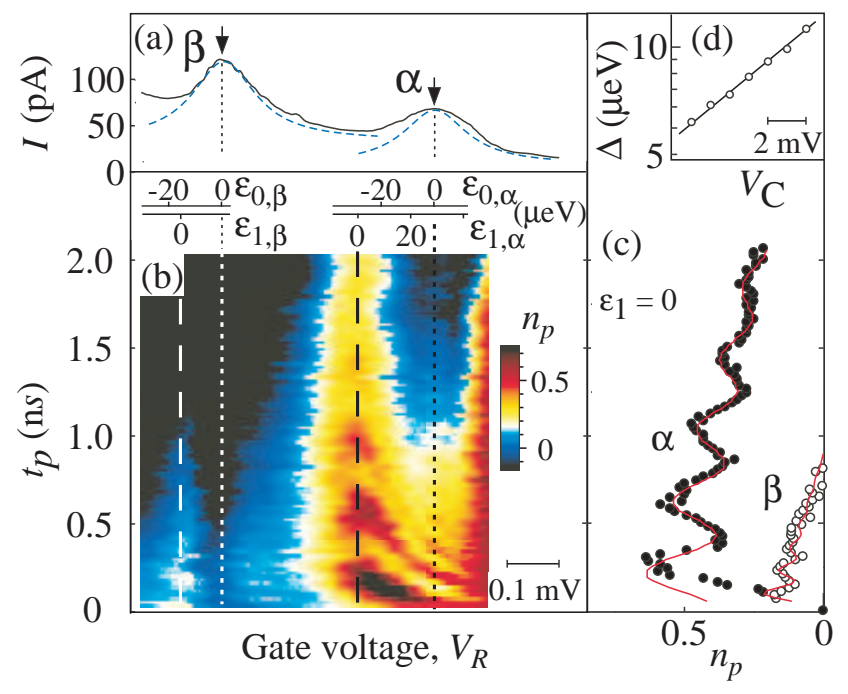

FIG. 2: (Color) (a) Current profile, $I$ vs $V_{R}$, at constant $V_{s d}=$ $650 \mu \mathrm{V}$. Two resonant tunneling peaks, $\alpha$ and $\beta$, out of about six peaks in the transport window, are shown. The ground state resonant peak (not shown) is located at about $0.5 \mathrm{mV}$ to the right of peak $\alpha$. Lorentzian fitting (dashed lines) to peaks $\alpha$ and $\beta$ gives approximate parameters $\hbar \Gamma_{L} \sim \hbar \Gamma_{R} \sim 30 \mu \mathrm{eV}$. (b) Color plot of $n_{p}$ as a function of $V_{R}$ and $t_{p}$. The horizontal axis is also shown in terms of $\varepsilon_{0, i}$ and $\varepsilon_{1, i}$ for resonance $i$ ( $\alpha$ or $\beta$ ). (c) $n_{p}\left(t_{p}\right)$ at $\varepsilon_{1}=0$ (long-dashed lines in b) for the resonance $\alpha$ (solid circles) and $\beta$ (open circles). Lines are fitted to the data. (d) The coupling energy, $\Delta$, determined from the oscillation frequency, when the gate voltage on $G_{C}$ is changed. The line is a guide for the eye.

$3(\mathrm{~b})]$, and the lattice temperature $T_{\text {lat }}[$ Fig. 3(c)]. Here $\Delta$, which is determined from the fitting ( $\hbar \Omega$ at $\varepsilon_{1}=0$ ), can be varied by changing the gate voltage $V_{C}$ on the central gate $G_{C}$ as shown in Fig. 2(d). Although decoherence from first-order tunneling processes is eliminated during manipulation, other decoherence sources are significant in our measurement.

Firstly, background charge fluctuations and noise in the gate voltages affect $\varepsilon$ and $\Delta$, which change the oscillation frequency $\Omega$ and dephase the system [9, 10]. The fluctuation, $\tilde{\varepsilon}$, of $\varepsilon$ in our sample ranges between $1.6 \mu \mathrm{eV}$, which is estimated from the fluctuation of $I$ in a frequency range $0.1-5 \mathrm{~Hz}$, and $3 \mu \mathrm{eV}$, which is the narrowest linewidth of the resonant peak we obtained in sample I in the weak coupling limit $(\Delta<1 \mu \mathrm{eV})$ [4]. The corresponding decoherence rate, $\Gamma_{\varepsilon}=|d \Omega / d \varepsilon| \tilde{\varepsilon}$ to the lowest order, for $\tilde{\varepsilon}=1.6 \mu \mathrm{eV}$ is shown by a solid line in Fig. 3(a). This qualitatively explains the large decoherence rate at $\varepsilon_{1} \neq 0$, where the system is sensitive to $\tilde{\varepsilon}$. However, the decoherence rate at $\varepsilon_{1}=0$ cannot be explained with this model, and should be dominated by other mechanisms.

Secondly, we consider cotunneling effects. Although the first-order tunneling processes are prohibited during manipulation, higher-order tunneling (cotunneling) 

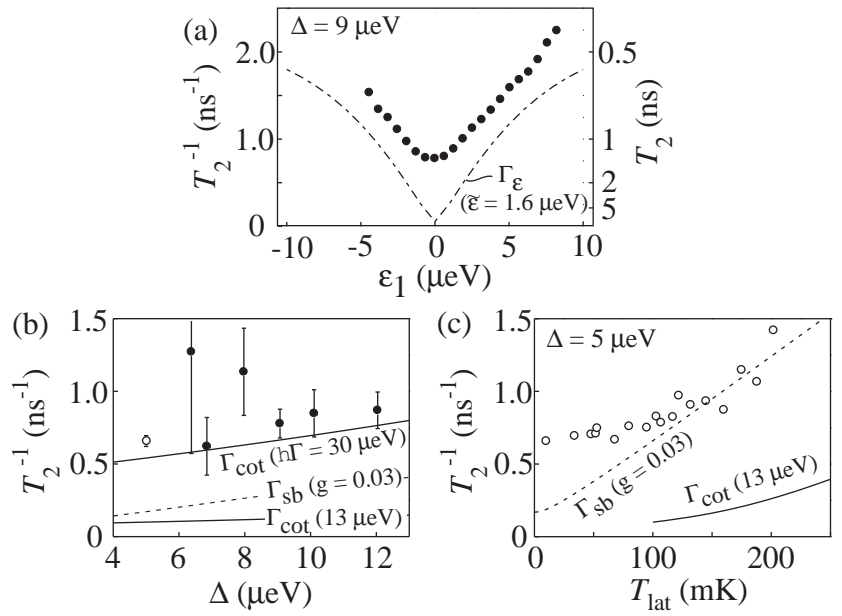

FIG. 3: Decoherence rate, $T_{2}^{-1}$, of the qubit. (a) The energy offset $\left(\varepsilon_{1}\right)$ dependence. The dash-dotted line shows the decoherence rate, $\Gamma_{\varepsilon}$, due to the fluctuation $\tilde{\varepsilon}=1.6 \mu \mathrm{eV}$. (b) The coupling energy $(\Delta)$ dependence. (c) The lattice temperature $\left(T_{\text {lat }}\right)$ dependence. The solid (open) circles were measured with $\hbar \Gamma_{L} \sim \hbar \Gamma_{R} \sim 30 \mu \mathrm{eV}$ in sample I $\left(\hbar \Gamma_{L} \sim \hbar \Gamma_{R} \sim 13\right.$ $\mu \mathrm{eV}$ in sample II). The decoherence rates calculated from cotunneling $\left(\Gamma_{c o t}\right)$ and spin-boson model $\left(\Gamma_{s b}\right)$ are shown by solid and dashed lines, respectively.

processes can occur because relatively high $\Gamma_{L}$ and $\Gamma_{R}$ were chosen for efficient initialization. For simplicity, we only estimate one of the cotunneling processes, which scatters the electron from the anti-bonding state to the bonding state (eigenstates of the qubit), from second order Fermi's golden rule. This gives a transition rate $\Gamma_{c o t}=(8 / h) \Delta(\hbar \Gamma)^{2} / U^{2}$ at $\varepsilon_{1}=0, V_{s d}=0$ and zero temperature when the barrier is symmetric $\left(\Gamma=\Gamma_{L}=\Gamma_{R}\right)$ 11]. $\Gamma_{\text {cot }}$ shown by solid lines in Figs. 3(b) and 3(c) actually includes thermal broadening in the source and drain $\left[T_{\text {elec }}=100 \mathrm{mK}\right.$ is assumed in Fig. 3(b)]. Although we cannot determine the parameters precisely, $\Gamma_{\text {cot }}$ is comparable to the observed $T_{2}^{-1}$. We believe that the cotunneling effect is significant in our measurement but can be easily diminished by choosing smaller $\Gamma$ and by making the interdot electrostatic coupling energy $U$ larger.

Lastly, we discuss electron-phonon interactions, which is an intrinsic decoherence mechanism in semiconductor QDs [4, 12]. Spontaneous acoustic phonon emission remains even at zero temperature and causes the inelastic tunneling between the two states [4]. The phonon emission rate estimated from the inelastic current or from the fitting with Eq. 22 is $\Gamma_{i} \sim(4-20 \mathrm{~ns})^{-1}$, which depends on $\Delta$, at $\varepsilon=-30 \mu \mathrm{eV}$. $\Gamma_{i}$ of our interest at $\varepsilon=0$ should be faster because of the spatial overlap of the eigenstates, and may be comparable to the observed $T_{2}^{-1}$. By assuming Ohmic spectral density for simplicity, the spin-boson model predicts the decoherence rate $\Gamma_{s b}=\frac{\pi}{4} g \Delta \operatorname{coth}\left(\Delta / 2 k_{B} T_{\text {lat }}\right)$ for $\varepsilon=0$, where $g=0.03$ is the dimensionless coupling constant that was chosen to fit with the temperature dependence data [see dashed line in Fig. 3(c)] 13, 14. This $g$ is a reasonable value to explain the inelastic current [13], and thus phonon emission seems to be significant in our system.

Therefore, the qubit is strongly influenced by lowfrequency fluctuation when $|\varepsilon| \gtrsim \Delta$, cotunneling at high tunneling rates, and acoustic phonons at high temperature. The resonances $\alpha$ and $\beta$ actually involve excited states in the right dot, and the relaxation to the ground state should also cause decoherence. Other mechanisms, such as the fluctuation of $\Delta$ and the electromagnetic environment, may have to be considered to fully understand the decoherence. It should be noted that the quality of the coherent oscillation was improved by reducing highfrequency noise from the gate voltages and the coaxial cable. The remaining noise may also contribute to the decoherence. We hope that some decoherence effects can be reduced by further studies.

In summary, we have successfully manipulated the artificial qubit in a double quantum dot. Coherent oscillations are observed for several combinations of ground and excited states. In the present experiments, there was no condition where two kinds of oscillations coincided, indicating that the two-level system is still a good approximation. However, application of a two-step voltage pulse, which consecutively adjusts the system at two resonances ( $\alpha$ and $\beta$ for instance) in a short time, would mix three quantum states coherently. Therefore, DQDs are promising for studying multi-level coherency [15], and the experiments can be extended to electron-spin manipulations and two-qubit operations [16].

We thank T. Brandes, T. Itakura, Y. Nakamura, K. Takashina, Y. Tokura, and W. G. van der Wiel for their stimulating discussions and help.

[1] M. A. Nielsen and I. L. Chuang, Quantum Computation and Quantum Information (Cambridge Univ. Press, Cambridge, 2000).

[2] S. Tarucha et al., Phys. Rev. Lett. 77, 3613 (1996).

[3] T. H. Oosterkamp et al., Nature 395, 873 (1998).

[4] T. Fujisawa et al., Science 282, 932 (1998).

[5] Y. Nakamura, Yu. A. Pashkin and J. S. Tsai, Nature 398, 786 (1999).

[6] W. G. van der Wiel et al., Rev. Mod. Phys. 75, 1 (2003).

[7] T. H. Stoof and Yu. V. Nazarov, Phys. Rev. B 53, 1050 (1996).

[8] T. Fujisawa et al., in preparation.

[9] T. Itakura and Y. Tokura, cond-mat/0303412 v1, to be published in Phys. Rev. B.

[10] Y. Nakamura et al., Phys. Rev. Lett. 88, 047901 (2002).

[11] M. Eto, Jpn. J. Appl. Phys. 40, 1929 (2001).

[12] T. Fujisawa et al., Nature 419, 278 (2002).

[13] T. Brandes and T. Vorrath, Phys. Rev. B66, 075341 (2002). 
[14] A. J. Leggett et al., Rev. Mod. Phys. 59, 1 (1987).

[15] T. Brandes and F. Renzoni, Phys. Rev. Lett. 85, 4148

[16] Yu. A. Pashkin et al., Nature 421, 823 (2003). (2000). 